\title{
Emprendimiento en ciudades intermedias en Chile: sus vínculos con el empleo y la sustentabilidad urbana
}

\section{Entrepreneurship in mid-sized cities in Chile: its links with employment and urban sustainability}

\author{
Sebastián Rodríguez 이 y Johannes Rehner² []
}

\begin{abstract}
RESUMEN
El emprendimiento se ha entendido como importante impulsor de nuevo empleo, y aporte al bienestar económico, por lo cual muchos programas de política pública se han enfocado en ello. Recurriendo a propuestas conceptuales que fundamentan esta supuesta relación, se argumenta aquí que el punto central para una evaluación desde la perspectiva de sustentabilidad radica en el empleo generado. El trabajo analiza cuantitativamente el reciente desarrollo del emprendimiento en diez ciudades de tamaño intermedio en Chile y el empleo asociado. Los resultados muestran que, si bien, el dinamismo del emprendimiento posee una temporalidad similar en las ciudades, existen diferentes pautas espaciales, destacando el mayor dinamismo en las ciudades del norte del país. Tanto la política de fomento al emprendimiento, como la existencia de liquidez financiera se ofrecen como explicación. Sin embargo, ello no se traduce de manera similar en la creación de nuevos puestos laborales, y además la estructura del empleo generado muestra debilidades, evidenciando así, que no se genera el esperado aporte - por lo menos desde una perspectiva de desarrollo urbano sustentable.
\end{abstract}

Palabras clave: emprendimiento, empleo, desarrollo urbano sustentable

\begin{abstract}
Entrepreneurship has been understood as an important driver of new employment, and of economic well-being, and therefore many public policy programs have focused on it. Drawing on conceptual proposals that underpin this supposed relationship, it is argued here that the central point for an assessment from a sustainability perspective lies in the employment generated. The paper analyzes quantitatively the recent development of entrepreneurship in ten medium-sized cities in Chile and the associated employment. It becomes evident that, although entrepreneurship has a similar temporality in the analyzed cities, there are different spatial patterns, highlighting the greater dynamism in northern cities. Both, the policy of encouraging entrepreneurship and the existence of financial liquidity, are offered as an explanation. However, this is not creating new jobs in a similar manner, and the created workplaces show structural weaknesses, evidencing that the expected contribution is not generated - at least not from a sustainable urban development perspective.
\end{abstract}

Keywords: entrepreneurship, employment, urban sustainable development

Centro de Desarrollo Urbano Sustentable. Pontificia Universidad Católica de Chile. 
Los autores agradecen los comentarios y sugerencias realizados por los evaluadores anónimos, que han contribuido a mejorar y enriquecer el manuscrito original. Trabajo financiando por la Agencia Nacional de Investigación y Desarrollo, Proyecto FONDAP Nº 15110020.

El emprendimiento es presentado frecuentemente como una "receta mágica" para alcanzar mayor crecimiento y desarrollo, especialmente en contextos del liberalismo económico. Notablemente, economías neoliberales hacen importantes esfuerzos no solamente para facilitar el emprendimiento, sino que también, lo fomentan con beneficios directos. Chile destaca a escala mundial por su alto nivel de emprendimiento, lo que algunos consideran un gran éxito (Espinoza et al., 2019). Sin embargo, muchos de las nuevas empresas tienen baja intensidad de conocimiento, por lo cual, el esperado impulso económico se relaciona principalmente con el empleo que estas son capaces de generar. Este efecto entonces estará en el centro del presente artículo. Desde una perspectiva del desarrollo sustentable urbano, planteamos que es el empleo digno lo que constituye el elemento clave para la "prosperidad" de las personas.

La amplia bibliografía europea sobre emprendimiento y su relevancia económica (Acs, 2016; Audretsch \& Fritsch, 2002; Brixy, 2014; Carree, 2003; Fotopoulos \& Spence, 1998; Schmude et al., 2008), contrasta con una escasez relativa de tales estudios en Chile - siendo un campo de desarrollo académico reciente (Amorós et al., 2013; Atienza et al., 2016; Espinoza et al., 2019). Estos trabajos agregan una mirada regionalmente diferenciada, ya que el emprendimiento muestra pautas espacio-temporales muy dispares (GEM, 2017). De allí nace la necesidad de estudios geográficos sobre el vínculo entre espacio y empleo, considerando especialmente las ciudades en regiones exportadoras, debido al auge que han vivido (Rehner, Rodriguez y Murray, 2018). Proponemos una discusión del dinamismo del emprendimiento y analizaremos en qué medida esto se traduce en diversificación productiva y mayor empleo, para indagar si puede favorecer a la sustentabilidad urbana.

Este artículo se divide en cinco secciones. Primero se plantean los principales argumentos teóricos, referidos al emprendimiento y su vínculo con el crecimiento económico, la política pública y la relación entre emprendimiento y sustentabilidad. Luego se describen algunas características estructurales del emprendimiento en Chile, seguido por la metodología. En la cuarta sección se muestran y discuten resultados, y finalmente, se plantean interpretaciones de la importancia del emprendimiento para el desarrollo urbano sustentable, incluyendo implicancias teóricas.

\section{El estudio del emprendimiento: su vínculo con el crecimiento y el empleo}

Es preciso discutir aspectos teóricos del vínculo entre emprendimiento, empleo y crecimiento, desde una perspectiva estructural, considerando el habitual reclamo a la política pública que fomente el emprendimiento nuevo (Thurik y Wennekers, 2004). Tal importancia política radica en la visión que, el emprendimiento es un elemento crucial para alcanzar crecimiento económico (Carree y Thurik, 2003) y aumentar la competitividad (Porter, 1990), aportando al bienestar económico de la sociedad (Audretsch et al., 2002). Desde una perspectiva de geografía económica con mira al desarrollo sustentable, resulta clave cuestionar estos impactos en el espacio. 
Primero, intuitivamente parece lógico que un mayor nivel de emprendimiento debería fomentar el crecimiento económico, porque aumenta la producción, genera ingreso y trabajo. La economía neoclásica fundamenta tal intuición, argumentando que el aumento del uso de los factores productivos trabajo y capital genera crecimiento (Solow, 1956). Además, desde que Schumpeter (1942) introduce el concepto "destrucción creativa", se ha enfatizado que el emprendimiento genera innovación y mejora la productividad de los factores - introduciendo investigación y conocimiento como categorías relevantes en la teoría de crecimiento (Romer, 1986).

Sin embargo, los numerosos estudios al respecto llegan a resultados ambiguos. Por un lado, se ha planteado que el emprendimiento es un aporte a la prosperidad a largo plazo (Ribeiro-Soriano, 2017:1), aumentando la tasa de empleo a nivel nacional e impulsando el crecimiento económico (Audretsch et al., 2002; Carree y Thurik, 2003), sobre todo cuando los emprendedores ponen en valor nuevas oportunidades (Acs 2006). Por otro lado, se ha argumentado que muchos emprendimientos, especialmente aquellos creados por necesidad (Acs 2006) carecen de innovación, y tienen un limitado efecto sobre el empleo, por lo cual se deduce que no producen riqueza (Shane, 2009). Las condiciones espaciales, necesidades, comportamiento, incentivos y el tipo de negocio son decisivos para el efecto sobre el crecimiento económico (Audretsch et al., 2002).

A pesar de ello, el fomento al emprendimiento tiene protagonismo en la política de desarrollo en Chile (Atienza et al., 2016), debido a la convicción que ayuda al desarrollo, lo que se hace relevante en dos escalas geográficas: a escala nacional apoyando una política pro-emprendimiento y a escala regional y local por la búsqueda de diversificación a través del emprendimiento, sobre todo en territorios dedicados a la exportación. Un ejemplo es la Estrategia Regional de Desarrollo de Antofagasta 2009 - 2020 que combina el reconocimiento de la "vocación minera", con la búsqueda de una mayor diversificación para lograr un entorno económico más estable (Gobierno Regional de Antofagasta, 2008). Notablemente se ha mostrado en Chile que, a pesar del centralismo, en las regiones chilenas no metropolitanas existe una mejor percepción del apoyo gubernamental al emprendimiento que en la capital (Amorós et al., 2013).

Respecto al mercado laboral el nivel de desempleo puede afectar la dinámica empresarial de distintos modos. Por un lado, el desempleo puede impulsar el emprendimiento, cuando personas desocupadas crean nuevos negocios por necesidad (Atienza et al., 2016). En Chile, el empleo independiente tiene una importancia elevada, sobre todo entre las personas sin formación profesional o técnica (OIT, 2018: 108) y se da frecuentemente por necesidad (Modrego, 2017). Pero una gran parte de los desocupados carecen de competencias empresariales y de capital (Calá, 2018), condiciones importantes para ser exitosos a largo plazo, como se ha mostrado en otros contextos de periferia (Brünjes \& Revilla Diez, 2013). Por otro lado, un alto nivel de desempleo es expresión de una condición macroeconómica desfavorable, lo que se puede traducir en un aumento del número de empresas que fracasan (Brixy y Grotz, 2007).

En esta misma línea, es relevante considerar las influencias coyunturales, especialmente de las crisis económicas. En una fase de crisis la "turbulencia" del emprendimiento aumenta: se crea un número significativo de empresas, pero, una parte importante de estas no perdura en el tiempo, produciendo la denominada "puerta giratoria" (Calá, 2018). Por otro lado, se ha mostrado que, si el emprendimiento se basa en la innovación, ha sido menos afectado por las crisis (Davidsson \& Gordon, 2016). En América Latina sin embargo, el emprendimiento por necesidad 
aumenta como efecto de grandes crisis y disminuye en fases favorables, por ejemplo en la fase de auge minero en Chile (Acs y Amorós, 2008: 317). Considerando que economías basadas en la extracción de recursos naturales, viven ciclos coyunturales muy marcados, donde el desempleo urbano es susceptible a las variaciones de precio de los minerales (Atienza et al., 2015), se puede suponer que personas que pierden su empleo a raíz de una crisis podrían buscar una salida en el emprendimiento, pero este tiende a ser pasajero, ya que en una fase próspera puede ser atractivo volver al empleo dependiente. Asimismo, en fases prósperas se genera una liquidez elevada, la cual requiere ser invertida para seguir produciendo renta (Rehner \& Rodriguez, 2017). Se esperaría entonces un nivel elevado de emprendimiento en fases prósperas, por la búsqueda de renta financiera a través de la inversión en nuevos negocios.

Uno de los principales aspectos estructurales a considerar es el tamaño de las empresas. Hasta la segunda mitad del siglo pasado, dominaba el argumento que las grandes empresas impulsan el crecimiento, crean la mayor parte del empleo y promueven el desarrollo económico, actuando como motor del progreso y permitiendo ventajas en cuanto a innovación (Schumpeter, 1942: 3; Audretsch \& Fritsch 2002: 114). Esto comienza a cambiar en las últimas décadas del siglo $X X$, cuando se plantea que las empresas pequeñas muestran un grado mayor de innovación, son creadoras de empleo y crecimiento económico (Rothwell, 1989; Acs et al., 1999). Sin embargo, la predominancia de las pequeñas y medianas empresas tiende hacia una mayor "turbulencia", mostrando distintos resultados sobre el crecimiento (Calá, 2018; Audretsch y Fritsch, 2002; Reynolds, 1999; Fotopoulos y Spence, 1998). Esto se traduce en diferentes políticas a escala subnacional (Audretsch y Fritsch, 2002): algunas regiones obtienen el resultado esperado en términos de empleo y crecimiento, a través de un mayor apoyo al stock de empresas existentes, protegiendo el empleo en las empresas ya existentes más que impulsar nuevos emprendimientos. Otras regiones lo obtienen con un fuerte fomento al emprendimiento nuevo, aunque esto signifique el cierre de empresas antiguas y una muy corta "sobrevivencia" de muchas de las nuevas empresas.

Otra dimensión es la composición sectorial del emprendimiento. Espacios con una economía diversificada, podrían promover la entrada de nuevas empresas y aumentar sus posibilidades de éxito como también el tiempo que estas perduren (Kosacoff y Ramos, 1999). Particularmente relevante para Chile es el efecto de la actividad extractiva-exportadora sobre el dinamismo económico local (Rehner y Rodríguez, 2018) y regional, ya que ciertas ciudades regionales muestran condiciones de auge bajo la influencia de la exportación minera (Rehner, Rodriguez \& Murray 2018). Basado en los efectos de consumo y de inversión de liquidez financiera producida por un sector en auge, se puede esperar que el sector minero actúe impulsando el emprendimiento especialmente en las economías urbanas del norte del país (Rehner y Rodríguez, 2017). Esto no se refiere tanto a la pregunta si se generan "cluster" o "enclaves" (Phelps et al., 2015), sino principalmente a efectos secundarios sobre economías urbanas.

Respecto al papel que juega el espacio en el surgimiento de nuevos negocios, especialmente en escala local y regional, se ha sostenido que, los factores regionales no tienen un efecto generalizado, sino que, las condiciones regionales pueden estimular la creación de empresas en algunos sectores y desalentarla en otros (Brixy, 2014: 1051). Aun así, las características regionales poseen gran relevancia en la explicación del emprendimiento (Sternberg, 2007) y en su posibilidad de persistir en el tiempo (Fritsch et al., 2006). Se ha puesto creciente énfasis en la consideración de la contextualidad socio-espacial (Trettin \& Welter, 2011), siendo que en geografía la mayor 
parte de la literatura reciente sobre emprendimiento nace de la investigación de los procesos de aglomeración e innovación produciendo variadas ramificaciones conceptuales (Méndez, 2002; Moulaert \& Farid, 2013; Markusen, 1996; Boschma \& Frenken, 2013).

Este cuerpo teórico en combinación con la tradición de los cluster (Porter, 1998) ha inspirado numerosos programas de política pública de desarrollo local. Pero estos trabajos han puesto énfasis en las comparaciones internacionales y en el análisis de grandes aglomeraciones urbanas, con poca atención a ciudades intermedias. Esto constituye un problema con respecto a la formulación de política pública en dos sentidos: aplicando propuestas teóricas de un ámbito económico muy distinto de manera descontextualizada y cambiando de escala geográfica sin adaptación conceptual. Por esta razón y por la condición actual del emprendimiento en Chile es importante discutir el impacto del emprendimiento en ciudades no metropolitanas desde la perspectiva de sustentabilidad.

\section{Emprendimiento, crecimiento y desarrollo urbano sustentable}

Sorprendentemente en la discusión sobre sustentabilidad la dimensión económica no es muy trabajada, y la bibliografía se enfoca principalmente en tres grandes tópicos para discutir el vínculo entre desarrollo sustentable y emprendimiento.

Primero se puede identificar un enfoque en la importancia de la creación de nuevas empresas para el surgimiento de innovaciones y tecnologías más sustentables, siguiendo la lógica de la "economía verde", de las "ciudades inteligentes" y numerosas variantes de la ingeniería ambiental (ej. Ferrara, 2015). Esto se enmarca implícitamente en una visión optimista sobre la capacidad de la innovación tecnológica y comercial para resolver los desafíos de la sustentabilidad (para una discusión crítica ver por ejemplo Kingston \& Cauvin, 2015). No solo reconoce nuevas tendencias como energías renovables, movilidad eléctrica, mayor eficiencia energética y uso eficiente de otros recursos, reciclaje en su relevancia para la sustentabilidad, sino lo valora como nuevos campos de negocio. Así, surgen conceptos como "ecopreneur" (Gibbs, 2009), o incluso una visión del emprendimiento como "panacea" para curar la falta de sustentabilidad (Hall et al., 2010) Tal visión se ha hecho común a partir de los años 1990 y engancha fácilmente con la tradición del vínculo conceptual entre emprendimiento e innovación. Pero estas propuestas no necesariamente son parte de una visión integral de sustentabilidad sino pretenden aportar a una transición hacia un cambiado sistema socio-técnico con un modo de producción y consumo más sustentable (Coenen et al., 2012).

Segundo, se ha planteado una discusión que vincula lo económico con la sustentabilidad de manera muy distinta, a través del concepto de "trabajo digno". Esto es un tema central en la visión de Naciones Unidas y de la OIT sobre la sustentabilidad, expresado en los Objetivos del Desarrollo Sustentable (ODS), particularmente en el ODS número 8: "Promover el crecimiento económico sostenido, inclusivo y sostenible, el empleo pleno y productivo y el trabajo decente para todos". Es notable que el criterio de empleo digno o empleo decente, que no se ha impuesto de mismo modo como el concepto de "desarrollo humano" (Sehnbruch et al 2015), es recuperado en la discusión sobre desarrollo sustentable. Importante es también, que uno de los apartados 
pertenecientes al ODS 8 hace explícitamente referencia al fomento del emprendimiento y de las pymes (8.3) como vehículos para un desarrollo sustentable. Este papel del emprendimiento para la sustentabilidad se basa en el empleo y menos en los aspectos tecnológicos. El concepto de "empresa sustentable" usado por la OIT se refiere a la relación con los empleados, la formación de capital humano, condiciones laborales, remuneración y derechos laborales (ILO, 2007). Sin embargo, se ha planteado críticamente que la traducción del ODS 8 en la Agenda 2030 ha sido marcado por una visión pro-negocio, disminuyendo el enfoque en el trabajador, convirtiendo el "empleo digno" en una promesa del crecimiento en vez de ser derecho humano (Frey, 2017). Especialmente para regiones en condición de "periferia", es dudoso si el emprendimiento puede ser un vehículo importante para la sustentabilidad en el sentido del ODS 8, considerando la abundancia del emprendimiento por necesidad discutida anteriormente. También el aporte de las pymes puede ser cuestionado por el bajo nivel de sueldo, la inestabilidad y también por normativas o prácticas laborales distintas.

Por último, se discute críticamente el valor del crecimiento económico para el desarrollo, planteando que la aspiración por un continuo crecimiento contradice al principio de la sustentabilidad y además es poco ético. Esta discusión retoma la tradición de los "límites de crecimiento" planteado en los años '70 (Meadows et al., 1972) y formula lo que hoy se conoce como desarrollo "post-crecimiento" o "de-crecimiento" (Blühdorn, 2017; Jackson, 2009), frecuentemente asociado a una visión crítica del consumismo o a un inminente fin del capitalismo (Spash, 2015, Blühdorn, 2017). Claramente este enfoque en la suficiencia como principio ético y en la renuncia al consumismo ha sido cuestionado por ser una visión desde el norte global, poco considerado con la realidad de países de menor ingreso, pero ha traído también propuestas desde el sur, por ejemplo, en el trabajo de Amartya Sen, que han destacado que el crecimiento económico tiene un valor netamente utilitario y no es un fin en sí mismo (Acosta, 2008). Una vertiente epistemológica distinta, ha sido formulada en América Latina y es conocida como principio del "buen vivir", el cual también cuestiona el valor del crecimiento, planteando que "la idea de crecimiento material sin fin podría culminar en un suicidio colectivo" (Acosta, 2008: 34). Evidentemente según esta visión un mayor emprendimiento no tiene ningún vínculo con el desarrollo (sustentable).

El trabajo presente se enmarca en lo que el Centro de Desarrollo Urbano Sustentable (CEDEUS) entiende por "desarrollo sustentable", definiendo esto como un "proceso a través del cual las comunidades florecen de forma armónica tanto en las generaciones actuales como en las futuras" (CEDEUS, 2019). Esta metáfora hace alusión al crecimiento, reconociendo su necesidad para la prosperidad de la sociedad, posicionándose en una lectura distinta a la visión de "post-crecimiento" (Blühdorn, 2017) y próximo al vínculo entre crecimiento y el trabajo digno como base de la sustentabilidad, tal como lo formula el ODS 8 del PNUD. En este sentido el empleo generado, su cantidad, calidad y estabilidad, es el criterio central en la discusión sobre la relación entre emprendimiento y sustentabilidad. Pero esta visión agrega por lo menos tres aspectos adicionales de relevancia para una comprensión de desarrollo sustentable que trasciende de manera decisiva el aspecto de crecimiento: Primero, con la metafórica referencia al "florecer" se añade una dimensión estética ${ }^{3}$, que deja en claro que no es el mero crecimiento lo que hace el desarrollo susten-

La Estética, como una rama de la Filosofía se dedica a la comprensión de "lo bello", por lo cual su presencia aquí representa una dimensión de sustentabilidad que va más allá del cumplimiento de indicadores acordados y consiste en el vínculo de los sujetos con su entorno, desde una perspectiva de calidad de vida. 
table sino lo decisivo resulta ser su aporte a la calidad de vida de las comunidades. Además, la metáfora hace alusión a la fertilidad y a lo cíclico de los procesos naturales de crecimiento - se puede interpretar esto como un reconocimiento que diferentes fases coyunturales se van alternando, pero se requiere que esto se traduzca en un crecimiento orgánico. Se suma la necesidad que este florecer sea armónico lo que agrega el criterio social más allá de la simple inclusión en el mercado laboral. Resulta fundamental relacionar la cantidad empleo generado por crecimiento con su característica, especialmente considerando las multidimensionales aproximaciones a "calidad de empleo" desarrollado últimamente, los cuales consideran de manera distinta precariedad de salario, contrato y seguridad social (Sehnbruch et al 2019, Fuentes \& Hernando 2019).

A raíz de estas reflexiones el trabajo presente se pregunta por el dinamismo del emprendimiento en espacios urbanos (no-metropolitanos) en Chile, sus implicancias estructurales (diversificación y estabilidad) y efectos en el empleo como insumo para la sustentabilidad urbana. Esto incluye la pregunta por las características de empleo en términos sectoriales, su estabilidad y nivel de ingreso.

\section{Nivel y características actuales del emprendimiento en Chile}

Chile destaca por su alto nivel de emprendimiento y creación de empresas nuevas, aspecto que se sostiene en la particularidad socio-cultural que en el país existe una notable intención de emprender. El Global Entrepreneurship Monitor (GEM) muestra que, en Chile, la población adulta con intención de emprender es de 47\% en 2017 (GEM, 2017), superando claramente a otros países de América Latina y el Caribe (en promedio 30\%); y de los países OCDE (en promedio 15\%), siendo Chile el país OCDE con el mayor nivel de intención de emprender (GEM, 2017). Además, este estudio muestra que en Chile la población cree que existe un ambiente propicio para emprender, y existe una confianza alta en el negocio nuevo (GEM, 2017).

Figura $\mathrm{N}^{\circ} 1$ :

Actividad emprendedora según tipo y comparación internacional (\%)

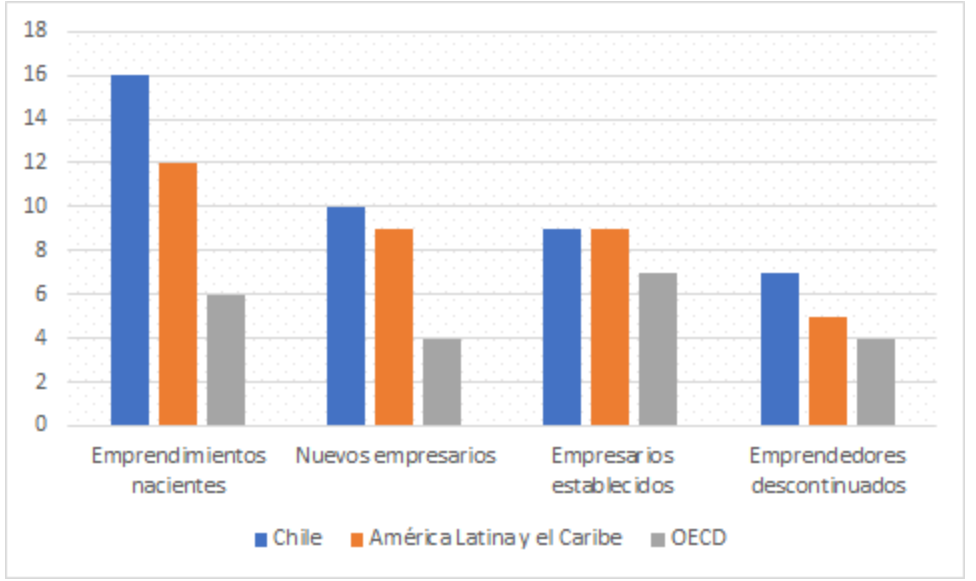

Fuente: Elaboración propia con base en GEM, 2018 
Respecto al ciclo de vida de los emprendimientos, en Chile destaca lo numeroso de los casos en la etapa de "emprendedores nacientes" (GEM, 2017: 39). Mientras tanto, el nivel de los emprendimientos establecidos que perduraron en el tiempo es similar al resto de los países. De esto se puede inferir un alto porcentaje fracasa en la fase inicial, lo que ilustra también la figura 1. Esto se relaciona con características estructurales e individuales de los emprendimientos, y pone en duda cuán beneficiosos pueden ser a largo plazo (Atienza et al., 2016).

Respecto a la motivación de emprender, destaca que en la fase inicial un 38\% emprende para aumentar sus ingresos, un $22 \%$ para incrementar su independencia, y un $31 \%$ indica hacerlo por necesidad (GEM, 2017: 44). Así, en Chile el porcentaje de emprendedores por necesidad es mayor que en los países OCDE (GEM, 2017: 46). Se debe entonces considerar que una parte relevante del emprendimiento en Chile es más respuesta de los individuos frente a problemas del mercado laboral que un motor de renovación de la actividad productiva en camino a una mayor productividad. Esto se condice también con la observación de un elevado nivel de auto - empleo en Chile, correspondiente unos $22 \%$ de la fuerza laboral, situación característica a nivel latinoamericano (OIT, 2018: 107-108).

En consecuencia, el dinámico emprendimiento no se refleja un impacto equivalente en la generación de trabajo: al contrario, un $54 \%$ de los emprendimientos nacientes en Chile manifiesta no haber creado empleos aún, mientras que el $46 \%$ restante menciona que ha creado entre 1 y 19 empleos (GEM, 2018). Tal limitado impacto en número de empleos se mantiene en el tiempo en muchos emprendimientos y una importancia limitada de las empresas chicas en el mercado laboral: Cerca del $30 \%$ de los emprendimientos establecidos manifiesta no haber creado empleos (GEM, 2018) y solamente un $7 \%$ de la fuerza laboral trabaja en empresas privadas con 5 o menos empleados (OIT, 2018: 108). Así, la expectativa de creación de empleos como consecuencia de la actividad emprendedora en el país resulta ser más optimista que la realidad actual.

\section{Metodología}

Este trabajo se basa en un análisis estadístico del número de empresas y trabajadores, diferenciado por sector económico, a escala ciudad y comprende los años 2005 - 2016. Los datos se obtuvieron a partir de los contribuyentes clasificados como personas jurídicas registrados en el Servicio de Impuestos Internos (SII). Se considera particularmente la creación de empresas, registrado como inicio de actividades en el SII. Además, se utilizó el número de trabajadores informados por cada empresa en el proceso tributario. Respecto a los sectores analizados, se diferenciaron empresas y trabajadores, según la clasificación CIIU. Esto permite identificar la especialización v/s diversificación económica de las ciudades, utilizando el Índice Hirschmann Herfindahl $(\mathrm{HHI})$.

Se trabajó con diez ciudades, cinco de ellas localizadas en el norte (lquique, Antofagasta, Calama, Copiapó y La Serena - Coquimbo) y otras cinco en el sur del país (Talca, Chillán, Concepción, Los Ángeles y Temuco), para así contrastar contextos espaciales diferentes. Esta 
selección se debe a que estas ciudades se localizan en territorios marcadas por la actividad exportadora de la minería en el norte y la triada papelera - forestal - agrícola en el sur. Cabe mencionar que, en base a la clasificación OCDE (2012), en algunos casos fue necesario realizar una agrupación de comunas como Concepción, mientras que, otras ciudades corresponden a una sola comuna, como es el caso de Antofagasta. Además, del listado de áreas urbanas funcionales propuesto por OCDE (2012), no se incluyó Arica (condición fronteriza) y Ovalle (se consideró a la conurbación La Serena - Coquimbo en esta región) de la zona norte del país, tampoco se consideró ciudades localizadas en las regiones de Valparaíso, Metropolitana y O'Higgins, debido a su cercanía con el área metropolitana de Santiago y capital nacional, y finalmente, para la zona sur, se consideró la inclusión de una ciudad en cada una de las regiones analizadas (Maule, Ñuble, Biobio y Araucanía).

Se realizaron dos test estadísticos de comparación de medias para corroborar la solidez de los resultados. El primero (t-test de Student) se basó en la corroboración de las diferencias en la dinámica del emprendimiento y empleo entre ciudades del norte y sur del país. Para ello, se consideró un conjunto de 50 datos en cada grupo, correspondiente a la tasa de crecimiento en cada una de los diez años de análisis, en cada una de las cinco ciudades en el norte, respectivamente en el sur. El segundo test se basó en la corroboración de las diferencias en relación a la política de fomento al emprendimiento. Para ello, se consideró un conjunto de 50 datos, correspondiente a cinco años de análisis entre 2006 y 2010, y luego, entre los años 2011 y 2015, por el conjunto de las diez ciudades analizadas. Para verificar lo anterior, se realizó una prueba no paramétrica, empleando el test $U$ de Mann-Whitney, obteniendo resultados similares y que confirman las diferencias que se plantean en los resultados.

\section{El emprendimiento urbano y sus efectos en el empleo}

El crecimiento del número de empresas entre 2006 y 2016 muestra dos fases con dinámicas muy distintas. Un primer período (hasta 2010) es caracterizado por un crecimiento estable y de moderada velocidad en contraste a la segunda fase (desde 2011), con crecimiento mucho más rápido en la mayoría de las ciudades. La velocidad de creación de emprendimientos nuevos muestra la misma tendencia (ver figura 2).

Sin embargo, el empleo asociado a estas empresas no muestra una pauta similar, lo que ilustra que el dinamismo en el emprendimiento no se traduce en un crecimiento similar en la creación de puestos laborales. Incluso, se evidencia una tendencia opuesta desde el año 2011 en adelante, cuando el fuerte aumento en el crecimiento del número de empresas coincide temporalmente con una leve reducción del crecimiento del empleo. La tendencia del crecimiento del empleo asociado a la creación de nuevas empresas varía mucho entre ciudades, es inestable y también registra una leve disminución en el período 2011 - 2014 (ver figura 3). En consecuencia se debe constatar que el aporte de emprendimiento al bienestar y la sustentabilidad urbana a través de puestos laborales es menos potente y además se caracteriza por ser inestable. 
Figura $\mathrm{N}^{\circ} 2$ :

Tasa de crecimiento de las empresas nuevas (\%)

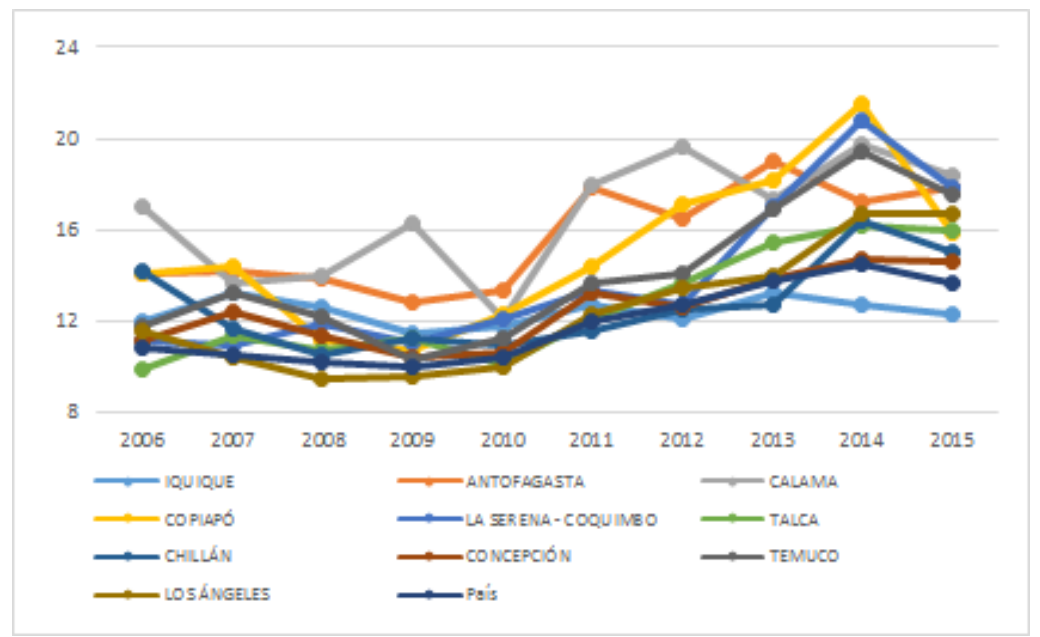

Fuente: Elaboración propia con base en SII, 2017

Figura $\mathrm{N}^{\circ} 3$ :

Tasa de crecimiento del empleo generado por empresas nuevas (\%)

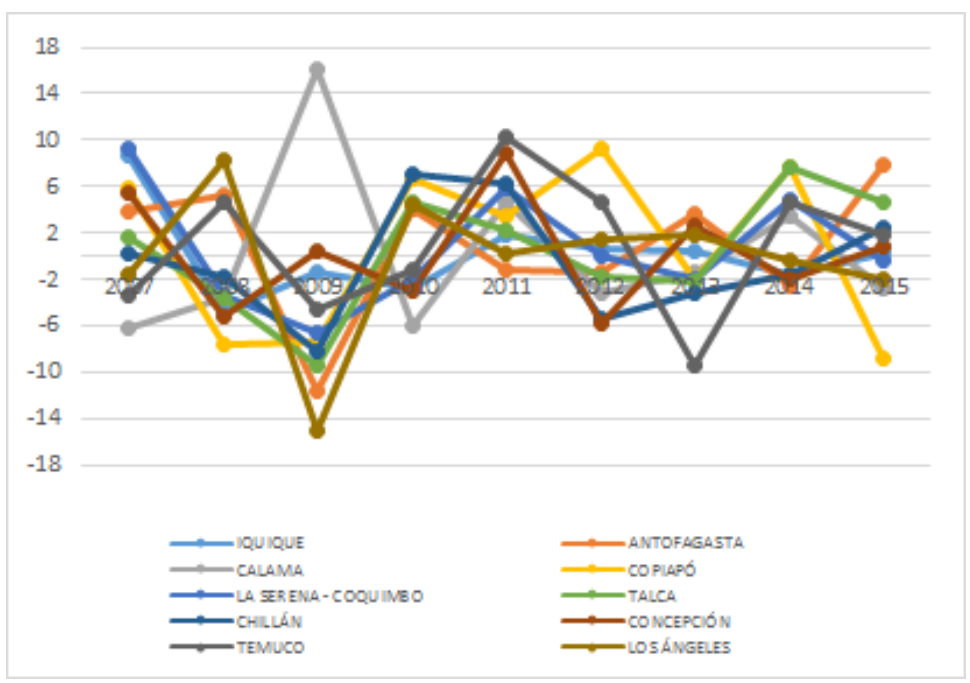

Fuente: Elaboración propia con base en SII, 2017

Interpretando ambas tendencias a partir del panorama económico durante la última década (Banco Central de Chile, 2019), es posible concluir que, la fase de auge exportador del cobre (hasta 2008) no coincide con un mayor crecimiento del número de empresas, pero sí con un mayor crecimiento del empleo. Luego, la crisis financiera global impacta de forma importante en el crecimiento del empleo. Por su parte, el crecimiento de las empresas parece no reaccionar de la misma forma a la situación de crisis económica externa, más bien, las ciudades muestran un nivel estable entre 2008 y 2009. Finalmente, la fase de recuperación de la exportación y precio del co- 
bre entre 2011 y 2012, está asociada a un nivel importante en el crecimiento de las empresas, pero no así en el empleo, el cual muestra una caída lenta pero sostenida en los últimos años.

Desde una perspectiva espacial, las ciudades del norte muestran un crecimiento del número y creación de nuevas empresas que supera al resto de las ciudades, especialmente desde 2011 en adelante. Para corroborar la solidez de este resultado, la tabla 1 muestra un test estadístico de comparación de medias en la creación de nuevas empresas y empleo entre las cinco ciudades del norte y sur del país. Se observa que las ciudades del norte muestran un mayor crecimiento de emprendimiento, argumento consistente con la discusión del auge generado en ciudades mineras y su impacto en otros sectores de la economía urbana (Rehner, Rodriguez \& Murray, 2018; Rehner \& Vergara, 2014; Rehner et al., 2019). Esto puede ser interpretado como una retroalimentación positiva, donde las ciudades con crecimiento fuerte tienden a una mayor creación de empresas, donde la inversión en construcción y el gasto en consumo fue dinámica y reaccionaba a mayores ingresos provenientes del sector minero. Respecto al crecimiento del empleo en empresas nuevas, sin embargo, no es posible identificar significativas diferencias entre las ciudades. El análisis espacial confirma entonces que el auge en emprendimiento no se traduce en similar efecto en empleo creado por ello.

Tabla $N^{\circ} 1$ :

Test de diferencia en creación de empresas y empleo por grupo de ciudades

\begin{tabular}{|l|l|r|r|r|r|}
\hline Ciudades & \multirow{2}{*}{$\begin{array}{l}\text { norte v/ sur } \\
\text { Norte }\end{array}$} & Media & $\begin{array}{c}\text { Desviación } \\
\text { estándar }\end{array}$ & $\begin{array}{c}\text { Media de } \\
\text { error estándar }\end{array}$ & Significancia \\
\hline Sur & Tasa de crecimiento de & 14,698 & 2,941 & 0,416 & 0,001 \\
\cline { 3 - 6 } & empresas nuevas & 12,831 & 2,387 & 0,338 & 0,001 \\
\hline Norte & Crecimiento del empleo & 1,005 & 0,057 & 0,008 & 0,744 \\
\cline { 5 - 6 } Sur & de nuevas empresas & 1,001 & 0,053 & 0,008 & 0,744 \\
\hline
\end{tabular}

Fuente: Elaboración propia, con base en SII, 2017

Agregando a esta diferencia espacial la visión temporal, es importante considerar que la política pública se ha enfocado en el fomento del emprendimiento, particularmente a partir de 2011, lo que parece estar asociado a los resultados obtenidos. Desde el inicio de esta década, el Estado ha instalado discursivamente el fomento del emprendimiento como mecanismo para el desarroIlo económico (Aravena et al., 2012). Una de las medidas fue resolver problemas en la etapa de creación y crecimiento de las empresas de menor tamaño, referidos principalmente a simplificar el marco regulatorio, destrabar y acelerar la creación. A partir de 2011, bajo un gobierno con marcada política pro-emprendimiento, cambios en el sistema de registro de nuevas empresas, disminuyeron el costo de registrar una empresa y la cantidad de días requeridos para este trámite, y en 2013 se aprueba una ley para crear sociedades en un día, y a costo cero. Según fuentes oficiales esto se tradujo en el crecimiento notable de sociedades creadas a partir del año 2011 (Ministerio de Economía, Fomento y Turismo, 2013).

Comparando estadísticamente los períodos 2006-2010 y 2011-2015, se muestra que la tasa de crecimiento de empresas nuevas es ampliamente superior en el segundo periodo (tabla 2). Aparentemente, la política pro-emprendimiento generó un número mayor de empresas nuevas. Por otra parte, no se observan diferencias significativas respecto al crecimiento del empleo. Apa- 
rentemente la política de fomento aumentó la tasa de emprendimiento, pero esto no se refleja en una aceleración de la creación de empleo. Por esto, la política pro-emprendimiento no tendría el efecto esperado sobre el empleo, limitando su capacidad de amortiguar futuras crisis.

Tabla $\mathrm{N}^{\circ} 2$ :

Creación de empresas y empleo por período de política de emprendimiento (\%)

\begin{tabular}{|c|c|c|c|c|c|}
\hline $\begin{array}{l}\text { Política de } \\
\text { emprendimiento }\end{array}$ & Variable & Media & $\begin{array}{c}\text { Desviación } \\
\text { estándar }\end{array}$ & \begin{tabular}{|c|} 
Media de \\
error estándar
\end{tabular} & Significancia \\
\hline $2006-2010$ & \multirow{2}{*}{$\begin{array}{l}\text { Tasa de crecimiento } \\
\text { de empresas nuevas }\end{array}$} & 11,972 & 1,652 & 0,234 & 0,000 \\
\hline $2011-2015$ & & 15,558 & 2,614 & 0,370 & 0,000 \\
\hline $2006-2010$ & \multirow{2}{*}{$\begin{array}{l}\text { Crecimiento del } \\
\text { empleo de nuevas } \\
\text { empresas }\end{array}$} & 0,994 & 0,065 & 0,010 & 0,154 \\
\hline $2011-2015$ & & 1,011 & 0,044 & 0,006 & 0,173 \\
\hline
\end{tabular}

Fuente: Elaboración propia con base en SII, 2017

Además, la política no tendría la característica de ser anticíclica, sino puede ser considerada en parte procíclica, ya que durante 2011 y 2012, período del auge en el emprendimiento, tiene lugar una fase de importante crecimiento de la exportación del cobre y, se puede suponer que parte de los excedentes obtenidos se invierten en nuevos emprendimientos. Posterior a la crisis subprime de 2009, se evidenció en el país un aumento considerable de los retiros de fondos de ahorro voluntario de pensión y una consecuente inversión por ejemplo en el sector inmobiliario (Rehner \& Rodríguez, 2017). Se podría suponer que, parte de estos ingresos y excedentes se hayan invertido en nuevos negocios y creación de nuevas empresas. Esta interpretación sólo tendría lugar desde el año 2011 en adelante y no así, en la fase inicial de auge exportador entre 2006 2008 , donde no se evidencia un crecimiento notorio del emprendimiento.

Otro argumento conceptual para responder al impulso del emprendimiento era la falta de oportunidades en el mercado laboral, quiere decir el emprendimiento por necesidad. En una economía dependiente de los ciclos de precio de commodities, como Chile, se podría esperar que las marcadas fases de auge y crisis impactan en el empleo e implícitamente en el empleo por necesidad. Sin embargo, la tasa de desempleo en las regiones donde se localizan las ciudades analizadas muestra un nivel relativamente estable durante la última década, con un ascenso a mediados de 2008 y finales de 2009, debido a la influencia indirecta de la crisis financiera global. No es posible encontrar relación entre un mayor desempleo y nivel de emprendimiento, lo que en parte se puede relacionar con escalas temporales y geográficas diferentes de los datos, por lo cual, el estudio presente no puede llegar a conclusiones con respecto a esta supuesta relación.

Por último, articulaciones pública-privada como por ejemplo CreoAntofagasta o Calama Plus se pueden interpretar como un mecanismo de implementación de política de fomento. La alianza pública-privada denominada Plan CREO Antofagasta, creada en 2012 y pensada con perspectiva hasta 2035 (CreoAntofagasta, 2017), involucra compañías mineras como BHP Billiton, Anglo American, Antofagasta Minerals, el sector público (Gobierno Regional y el Municipio Antofagasta), la academia, la comunidad, entre otros. Si bien, el objetivo auto-declarado de este programa radica en enfrentar los desafíos del crecimiento de Antofagasta mejorando la calidad de vida (CreoAntofagasta, 2017), se ha criticado que representa intereses económicos, principalmente de la gran 
minería (Bustos et al. 2019). Uno de los ejes de CREO Antofagasta hace referencia al fomento del emprendimiento ("AntofaEmprende"), y se puede suponer que, en parte, el surgimiento de nuevas empresas en estas economías podría estar asociado al desarrollo de planes y estrategias como CREO Antofagasta o Calama Plus, impulsando el emprendimiento en diferentes sectores económicos como parte de una agenda que busca en primer lugar crecimiento económico.

\section{¿El emprendimiento como mecanismo de solidez de las economías locales?}

Considerando lo anterior e intentando conocer si el emprendimiento se daría por la búsqueda de mayor diversificación en la estructura productiva de estas ciudades, identificamos las actividades con mayor emprendimiento y se evalúa si hay creciente concentración en determinados rubros, llevando a una especialización de estas economías locales. El conjunto de ciudades muestra en general una estructura relativamente diversificada del emprendimiento. La ciudad de lquique constituye una excepción, superando claramente el resto de las ciudades durante todo el periodo - posiblemente debido a la influencia particular de la Zona Franca (ZOFRI). Sin embargo, en los últimos años, todas las otras ciudades muestran un rápido aumento en el nivel de especialización, siendo la creación de nuevas empresas enfocada en sólo algunos sectores.

Respecto al grado de especialización del empleo generado, el conjunto de ciudades muestra mayor variabilidad y un nivel de diversificación menor que la creación de empresas. Esto puede ser interpretado en que, las empresas que surgen en estas ciudades responden a una demanda más bien diversificada, mientras que, el empleo tiende a concentrarse en determinados sectores económicos con una mayor intensidad laboral. El comercio $(24,2 \%$ de las empresas $/ 17,1 \%$ del empleo), la construcción (14,1\% / 27,3\%), y las actividades inmobiliarias, empresariales y alquiler $(19,3 \%$ / 15,5\%) superan en magnitud al resto de los sectores entre 2005-2015 tanto en creación de empresas como de empleo. Tal pauta ha sido discutida como un efecto secundario del auge exportador, causando el dinamismo en ciertos rubros, asociado al consumo y la inversión de liquidez financiera, impulsando actividades como la construcción y comercio (Corden y Neary, 1982; Rivera y Aroca, 2014; Rehner \& Vergara, 2014). Para el caso de las ciudades en auge del norte chileno, la dinámica en estos rubros podría ser reflejo de cómo los ingresos provenientes del cobre pueden difundirse y circular por las economías urbanas. Finalmente, cabe mencionar que, en ambos casos, existe una tendencia hacia una mayor especialización en los últimos años, lo que significa que el posible impulso diversificador, esperado del emprendimiento, está actualmente disminuyendo. 
Figura $\mathrm{N}^{\circ} 4$ :

Concentración sectorial de las empresas nuevas $(\mathrm{HHI})$

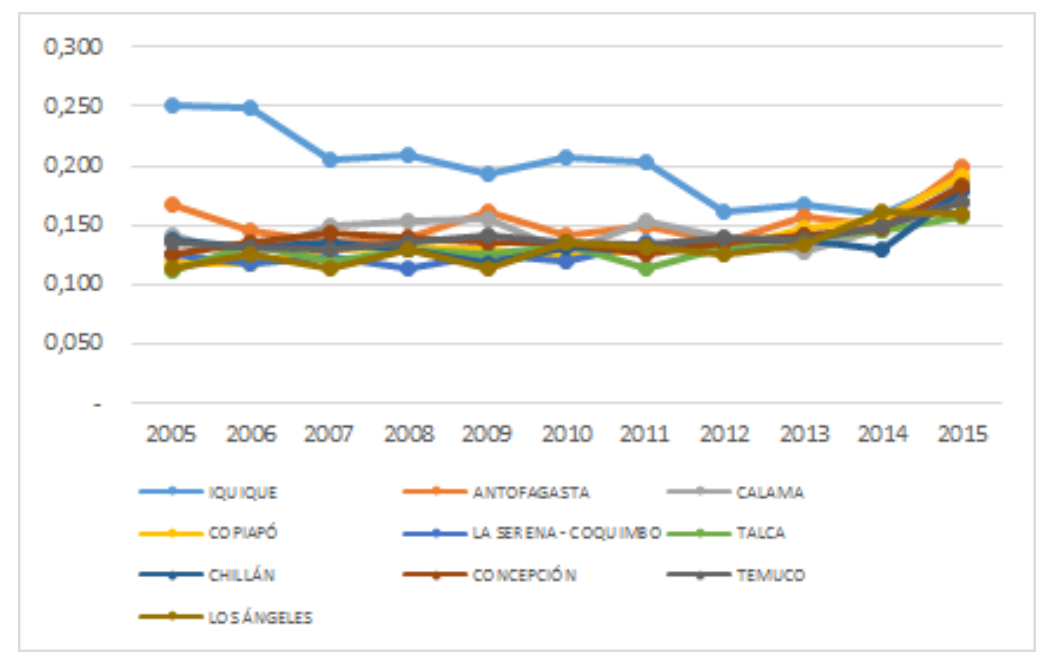

Fuente: Elaboración propia con base en SII, 2017

Figura $\mathrm{N}^{\circ} 5$ :

Concentración sectorial del empleo generado por empresas nuevas $(\mathrm{HHI})$

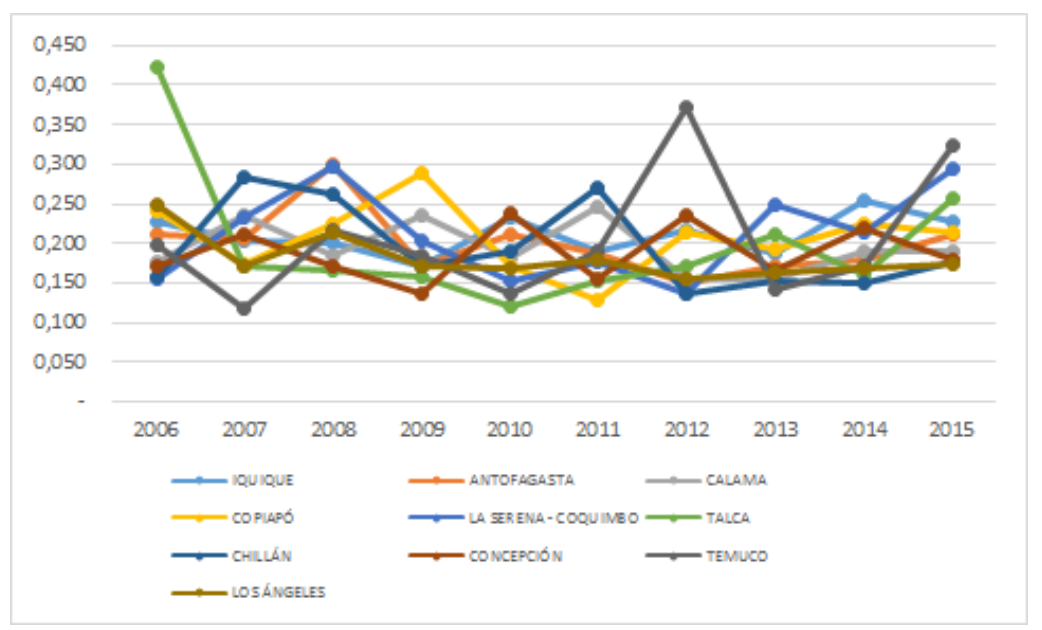

Fuente: Elaboración propia con base en SII, 2017

La tendencia de crear nuevos puestos laborales en comercio y construcción genera dudas con respecto a la sustentabilidad urbana desde una perspectiva de la calidad de los trabajos creados. Los sueldos en construcción y sobre todo en el comercio tienden a ser relativamente bajos (según encuesta CASEN) y por ende si bien a través del emprendimiento se genera empleo e implícitamente ingreso, una parte importante de ellos no provee bienestar económico relevante. A esto se suma que el comercio muestra una frecuencia de trabajos part-time en la categoría "subempleo" levemente elevada (Fuentes \& Hernando 2019: 7). Además el sector construcción muestra menor porcentaje de contratos indefinidos (según encuesta CASEN) y a se suma evidencia que demues- 
tra la particular problemática del empleo precario en el sector de construcción en principales centros urbanos de Chile, donde, esto se combina con mecanismos de subordinación en migrantes internacionales (Stefoni et al., 2017).

Por ende la concentración de la creación de empresas y empleo en estos sectores es preocupante desde la perspectiva del desarrollo sustentable, por una combinación de elementos. Primero, de acuerdo a la perspectiva de sustentabilidad aplicada en este trabajo, hay que constatar que si el emprendimiento implica un aumento de empleos asociados a rubros de limitado beneficio económico, baja seguridad social, alta susceptibilidad a los ciclos económicos externos, es dudoso en cuanto a su aporte a la promoción del trabajo digno. Segundo, emprendimiento y empleo que se ve impulsado principalmente por un aumento del consumo y de la edificación, evidentemente iría en contra de la concepción de un desarrollo sustentable basado en la suficiencia en el sentido de un desarrollo "post-crecimiento". Y, finalmente, tal tendencia evidentemente no se relaciona con un aporte relevante al desarrollo sustentable desde la perspectiva y tradición de la economía verde. Bajo cualquiera de las tres lecturas sobre el vínculo entre emprendimiento y desarrollo sustentable, la tendencia observada no parece aportar de manera relevante.

\section{Conclusiones}

La discusión anterior se centró en conocer la dinámica del emprendimiento en Chile, específicamente a escala urbana, mostrando diferencias temporales y espaciales en las ciudades analizadas. Una primera fase entre 2006 y 2010 se caracteriza por un crecimiento estable y de moderada velocidad, en contraste con la segunda fase, caracterizada por un crecimiento dinámico en la mayoría de las ciudades analizadas. Esto, se vincularía al impulso de la política pública pro-emprendimiento, donde el Estado, y en especial el gobierno de turno de aquel periodo, con una ideología pro-emprendimiento, ha apoyado la creación de nuevas empresas, bajo la idea de que el emprendimiento sea un mecanismo de crecimiento para el país.

Desde la perspectiva espacial, destaca el mayor dinamismo en las ciudades del norte del país. Posiblemente se asocia con la mayor liquidez que viven estas ciudades, donde la inversión en construcción y el consumo son dinámicos y reaccionan a mayores ingresos provenientes del sector extractivo-exportador. Complementando ello, es que la alianza público - privada también actuaría impulsando en parte el emprendimiento, destacando el rol que desarrollan las empresas mineras, por ejemplo, en el caso de Antofagasta.

Sin embargo, un mayor nivel de emprendimiento no se asocia necesariamente con nuevos puestos de trabajo. Al respecto, la tendencia temporal del empleo asociado a la creación de empresas posee una pauta diferente, incluso opuesta desde 2011. Ello, permite concluir que, la política pro emprendimiento no sería muy efectiva desde la perspectiva de crear empleo.

Así, resulta cuestionable cómo el emprendimiento puede ser un aporte para la sustentabilidad. Bajo la concepción teórica de sustentabilidad urbana, el análisis permitió concluir que, el fomento al emprendimiento como mecanismo para crear empleo no sería efectivo, y, por ende, se pone en duda si aporta a mayor prosperidad y bienestar de la población. Además, el emprendimiento se 
concentra en los rubros de comercio, construcción y actividades de alquiler, por lo cual, se pudo sostener que las ciudades analizadas poseen una estructura poco diversificada, concentrándose la actividad emprendedora y en especial, la creación de empleo, en estos tres sectores. Esto resulta aún más cuestionable, al considerar la calidad del empleo creado precisamente en estos sectores, caracterizado por bajos sueldos y contratos menos estables.

De esta forma, la dinámica del emprendimiento en estas ciudades, muestra una lógica que apuntaría al aumento del consumo y de la edificación, pero con limitado impacto en empleo y prosperidad. Ello estaría en tensión con la concepción de un desarrollo sustentable y, por ende, en los términos analizados aquí, el dinámico emprendimiento parece no aportar de manera relevante al desarrollo de la sustentabilidad urbana en las ciudades del país.

\section{Referencias}

Acosta, A. El buen vivir, una oportunidad por construir. Revista Ecuador Debate, 2008, No 75, p. 33-48.

Acs, Z. How is entrepreneurship good for economic growth? Innovations: Technology, Governance, Globalization, 2006, Vol 1, No 1, p. 97-107

Acs, Z. J. \& Amorós, J. Entrepreneurship and competitiveness dynamics in Latin America. Small Business Economics, 2008, No 31, p. 305-322 DOI 10.1007/s11187-008-9133-y

Acs, Z.J.; Carlsson, B. \& Karlsson, K. Entrepreneurship, Small and Medium-sized Firms and the Macroeconomy. Cambridge University Press, 1999.

Aravena, M.; Fara, C., \& Torres, V. Creación de una herramienta de diagnóstico para la micro y pequeña empresa. (Tesis para optar al título de Ingeniero Comercial, mención Administración Pública). Facultad de Economía y Negocios, Universidad de Chile. 2012. Recuperado el 22 de septiembre de 2017 desde: http://repositorio.uchile.cl/handle/2250/108118

Atienza, M.; Lufín, M. \& Romaní, G.: Un análisis espacial del emprendimiento en Chile. Más no siempre es mejor. Eure, 2016, Vol. 42, № 127, p. 111-135.

Atienza, M.; Lufin, M.; Soto, J. \& Cortés, Y. ¿Es la región de Antofagasta un caso exitoso de desarrollo local basado en la minería? En C. Rodríguez, M. Atienza, M. Lufin, G. Romaní, J. A. González, R. González, J. Soto, Sistemas, coaliciones, actores y desarrollo económico territorial en regiones mineras. Innovación territorial aplicada, 2015, (pp. 97-117). Antofagasta: Universidad Católica del Norte.

Amorós, J.; Felzensztein, C. \& Glimmon, E. Entrepreneurial Opportunities in Peripheral versus Core Regions in Chile. Small Business Economics, 2013, No 40, p. 119-139. doi:10.1007/s11187-011-9349-0.

Audretsch, D.B. \& Fritsch, M. Growth Regimes over Time and Space. Regional Studies, 2002, Vol. $36, \mathrm{~N}^{\circ} 2$, p. $113-124$. 
Banco Central de Chile. Base de Datos Estadísticos. Exportaciones de Bienes en millones de dólares FOB. 2019. Recuperado el 23 de julio de 2019 desde: https://si3.bcentral.cl/Siete/secure/ cuadros/arboles.aspx?idCuadro=BP6M_EXPORT

Blühdorn, I. Post-capitalism, post-growth, ¿postconsumerism? Eco-political hopes beyond sustainability, Global Discourse, 2017, Vol. 7, No1, p. 42-61, DOI: 10.1080/23269995.2017.1300415

Boschma, R.A. \& Frenken, K. ¿Por qué la geografía no es una ciencia evolutiva? Hacia una Geografía Económica Evolutiva. En: M. Valdivia López, J. Delgadillo Macías (ed.) La Geografia y la Economía en sus vínculos actuales. UNAM: Mexico D.F., 2013, p. 285-316.

Brixy, U. \& Grotz, R. Regional patterns and determinants of birth and survival of new firms in Western Germany, Entrepreneurship \& Regional Development, 2007, Vol. 19 N4, p. 293-312.

Brixy, U. The Significance of Entry and Exit for Regional Productivity Growth, Regional Studies, 2014, Vol. 48, N6, p. 1051-1070.

Brünjes, J. \& Revilla-Diez, J. "'Recession Push' and 'Prosperity Pull' Entrepreneurship in a Rural Developing Context." Entrepreneurship and Regional Development, 2013, Vol. 25, ํ3-4, p. 251-271. doi:10.1080/08985626.2012.710267.

BUSTOS GALLARDO, B.; LUKAS, M.; STAMM, C.; TORRE, A. Neoliberalismo y gobernanza territorial: propuestas y reflexiones a partir del caso de Chile. Revista de Geografia Norte Grande, 2019, n.73, pp.161-183.

Calá, C.D. Factores sectoriales y regionales que determinan la dinámica empresarial en los países en desarrollo: evidencia sobre las actividades manufactureras de tecnología baja, media y alta en Argentina. Revista Cepal, 2018, №124, p. 131 - 154.

Carree, M. A. \& Thurik, A. R. The impact of entrepreneurship on economic growth. In Z. J. Acs \& D. B. Audretsch (Eds.), Handbook of entrepreneurship research. Boston, MA: Kluwer Academic Publishers, 2003, p. 437-471. doi:10.1007/0-387-24519-7_17

CEDEUS. Centro de Desarrollo Urbano Sustentable. 2019. Recuperado el 02 de julio de 2019 desde: https://www.cedeus.cl/sobre-nosotros/el-centro/

Coenen, L.; Benneworth, P. \& Truffer, B. Toward a spatial perspective on sustainability transitions. Research Policy, 2012, N41, p. 968- 979.

Corden, W. \& Neary, J. Booming Sector and De-Industrialization in a Small Open Economy. The Economic Journal, 1982, Vol. 92, N³68, p. 825-848

CREO Antofagasta. Programa AntofaEmprende. 2017, Recuperado el 22 de mayo de 2019 desde: http://creoantofagasta.cl/ 
Davidsson, P. \& S. R. Gordon. "Much Ado about Nothing? The Surprising Persistence of Nascent Entrepreneurs through Macroeconomic Crisis." Entrepreneurship, Theory and Practice, 2016, Vol. 40, N4, p. 915-941. doi:10.1111/etap.12152.

Espinoza, C.; Mardones, C.; Sáez, K. \& Catalán, P. Entrepreneurship and regional dynamics: the case of Chile, Entrepreneurship \& Regional Development, 2019, DOI: 10.1080/08985626.2019.1565421

Ferrara, R. The Smart City and the Green Economy in Europe: A Critical Approach. Energies, 2015, Vol. 8 No 6, p. 4724-4734. DOI: 10.3390/en8064724

Fotopoulos, G. \& Spence, N. Entry and exit from manufacturing industries: symmetry, turbulence and simultaneity - some empirical evidence from Greek manufacturing industries, 1982-1988, Applied Economics, 1998, N³0, p. 245-262.

Frey, D.F. Economic growth, full employment and decent work: the means and ends in SDG 8 . The International Journal of Human Rights, 2017, Vol. 21, № 8, p. 1164-1184, DOI:10.1080/13642987.20 17.1348709

Fritsch, M.; Brixy, U. \& Falck, O. The Effect of Industry, Region, and Time on New Business Survival - A Multi-dimensional Analysis. Review of Industrial Organisation, 2006, Vol. 28, N³, p. 285-306

Fuentes, A. \& Hernando, A. Midiendo la calidad del empleo en Chile. Puntos de referencia Octubre $2019 N^{\circ}$. 521. Centro de Estudios Públicos. www.cepchile.cl

GEM. Reporte Nacional de Chile 2017, Recuperado el 10 de junio de 2019 desde: https://negocios. udd.cl/gemchile/files/2018/06/GEM_2017_final.pdf

GEM. Reporte Nacional de Chile 2018, Recuperado el 10 de junio de 2019 desde: https://negocios. udd.cl/gemchile/files/2019/06/GEM-Reporte-Nacional-de-Chile-2018-1.pdf

Gibbs, D. Sustainability Entrepreneurs, Ecopreneurs and the Development of a Sustainable Economy. Greener Management International, 2009, N55, p. 63-78.

Gobierno Regional de Antofagasta. Estrategia Regional de Antofagasta 2009 - 2020, 2008, Recuperado el 15 de mayo de 2019 desde: https://www.opia.cl/static/website/601/articles-40569_archivo_02.pdf

Hall, J.K.; Daneke, G.A. \& Lenox, M.J. Sustainable development and entrepreneurship: Past contributions and future directions. Journal of Business Venturing, 2010, N²5, p. 439-448

ILO. Conclusions concerning the promotion of sustainable enterprises. International Labour Conference, Geneva: International Labour Office. 2007.

INE. Encuesta Nacional de Empleo - ENE. 2019. Recuperado el 12 de junio de 2019 desde: https:// www.ine.cl/estadisticas/laborales/ene 
Jackson, T. Prosperity without Growth: Economics for a Finite Planet. London: Earthscan. 2009.

Kingston, R.; Cauvain J. Smart cities and green growth: Outsourcing democratic and environmental resilience to the global technology sector. Environment and Planning A, 2015, Vol. 46, No. 4, p. 803 - 819. DOI: 10.1068/a46242

Kosakoff, B. \& Ramos, A. The industrial policy debate. CEPAL Review, 1999, №68, p. 35-60.

Markusen, A. Sticky Places in Slippery Space: A Typology of Industrial Districts. Economic Geography, 1996, Vol. 72, N³, p. 293-313.

Meadows, D.H.; Meadows, D.L.; Randers, J. \& Behrens W.W. The limits to growth. Universe Books: New York. 1972.

Méndez, R. Innovación y desarrollo territorial: algunos debates teóricos recientes Revista eure, 2002, Vol. XXVIII, No 84, p. 63-83

Ministerio de Economía, Fomento y Turismo. Creación de Empresas: Una comparación internacional. División de Estudios. 2013, p. 1 - 12

Modrego, F.; Paredes, D. \& Romaní, G. Individual and place-based drivers of self-employment in Chile, Small Business Economics, 2017, №. 49, p. 469-492. DOI 10.1007/s11187-017-9841-2.

Moulaert, F. \& Farid, M. Modelos territoriales de innovación: una revisión crítica. En: Valdivia López, M. \& Delgadillo Macías, J. (Ed.) La Geografía y la Economía en sus vínculos actuales. Cuernavaca: UNAM. 2013, p. 439-460.

OIT. Panorama Laboral 2018. Lima. Organización Internacional del Trabajo. 2018.

Organization for Economic Co-operation and Development. Working party on territorial policy in urban areas. National urban policy reviews. The case of Chile. París: OECD Publishing. 2012.

Phelps, N.A.; Atienza, M. \& Arias M. Encore for the Enclave: The Changing Nature of the Industry Enclave with Illustrations from the Mining Industry in Chile. Economic Geography, 2015, Vol. 91, No2, p. 119-146.

Porter, M. Clusters and the new economics of competition. Harvard Business. Review NovemberDecember, p. 77-90. 1998.

Porter, M. E. The Competitive Advantage of Nations, New York: Free Press. 1990.

Rehner, J.; Rodriguez, S.; Murray, W. "Ciudades en auge en Chile: rol de la actividad exportadora en la dinámica del empleo urbano". EURE, 2018, Vol. 44, № 131, p. 151-171. 
Rehner, J.; Murray, W.E.; Rodríguez, S., \& Overton, J. Boom city! Regional resource peripheries and urban economic development in Chile, Area Development and Policy, 2019, Vol. 5, № 3, p. 305-323

Rehner, J., \& Rodríguez, S. La máquina de crecimiento en una ciudad minera y el papel del espacio público: el proyecto Parque Kaukari, Copiapó. Revista de Urbanismo, 2018, Nº 38, p. 1-22.

Rehner, J. \& Rodríguez, S. Inversión inmobiliaria en tiempos de auge y crisis ¿Es la ciudad un producto minero o un derivado financiero? Revista de Geografía Norte Grande, 2017, 67, p. 183-210.

Rehner, J.; Vergara, F. Efectos recientes de la actividad exportadora sobre la reestructuración económica urbana en Chile. Revista de Geografía Norte Grande, 2014, 59, p. 83-103.

Reynolds, P.D. ¿Creative destruction: source or symptom of economic growth?, in Acs, Z.J., Carlsson, B.\& Karlsson, Ch. (eds). Entrepreneruship, Small and Medium-Sized Enterprises and the Macroeconomy, Cambridge: Cambridge University Press, 1999, p. $97-136$.

Ribeiro-Soriano, D. Small business and entrepreneurship: their role in economic and social development, Entrepreneurship \& Regional Development, 2017, Vol. 29, №1-2, p. 1-3.

Rivera, N. \& Aroca, P. Escalas de producción en economías mineras. El caso de Chile en su dimensión regional. EURE, 2014, Vol. 40, №121, p. $247-270$.

Romer, P.M. Increasing Returns and Long-Run Growth. Journal of Political Economy, 1986, Vol. 94, N5, p. $1002-1037$.

Rothwell, R. Small firms, innovation and industrial change. Small Business Economics, 1989, Vol. 1, No1, p. 51-64.

Schmude, J.; F. Welter, \& S. Heumann. Entrepreneurship research in Germany. Entrepreneurship Theory and Practice, 2008, N³2, p. 289-311.

Schumpeter J. Capitalism, Socialism and Democracy. London. Routledge 1942.

SII - Servicio de Impuestos Internos (2017). Estadísticas y Estudios SII: Nómina de empresas personas jurídicas AT 2006 - 2016. Recuperado el 10 de junio de 2018 desde: http://www.sii.cl/estadisticas/nominas/nominapersonasjuridicas.htm

Sehnbruch, K., Burchell, B., Agloni, N., \& Piasna, A. Human development and decent work: why some concepts succeed and others fail to make an impact. Development and Change, 2015, 46(2), 197-224

Sehnbruch, K. González, P. Apablaza, M., Méndez, R. \& Arriagada, V. The Quality of Employment (QoE) in nine Latin American countries: A multidimensional perspective. World Development 127. 2019. https://doi.org/10.1016/j.worlddev.2019.104738 
Shane, S. Why encouraging more people to become entrepreneurs is bad public policy. Small Business Economics, 2009, Vol. 33, N², p. 141-149.

Solow, R. A contribution to the theory of economic growth. Quarterly Journal of Economics, 1956, No70, p. 65-94

Spash, C.L. The Future Post-Growth Society. Review Essay. Development and Change, 2015, Vol. 46, No2, p. 366-380. DOI: 10.1111/dech.12152

Stefoni, C.; Leiva, S. \& Bonhomme, M. Migración internacional y precariedad laboral. El caso de la industria de la construcción en Chile. Revista Interdisciplinar da Mobilidade Humana, 2017, Vol. 25, N49, p. $95-112$.

Sternberg, R. Entrepreneurship, proximity and regional innovation systems. Tijdschrift voor Economische en Sociale Geografie, 2007, №98, p. 652-666.

Thurik, R. \& Wennekers, S. Entrepreneurship, Small Business and Economic Growth." Journal of Small Business and Enterprise Development, 2004, Vol. 11, ํ1, p. 140-149.

Trettin, L. \& Welter, F. Challenges for spatially oriented entrepreneurship research, Entrepreneurship \& Regional Development, 2011, Vol. 23, N7-8, p. 575-602. 
